Technological University Dublin

DƯBLIN

ARROW@TU Dublin

1994-01-01

\title{
On Endomorphisms and Automorphisms of Some Pure Subgroups of the Baer-Specker Group
}

\author{
A. L.S. Corner \\ Technological University Dublin \\ Brendan Goldsmith \\ Technological University Dublin, brendan.goldsmith@tudublin.ie
}

Follow this and additional works at: https://arrow.tudublin.ie/scschmatart

Part of the Mathematics Commons

\section{Recommended Citation}

Corner, A.L.S. \& Goldsmith, B. On endomorphisms and automorphisms of some pure subgroups of the Baer-Specker group. Contemporary Mathematics, vol.171, pg. 69-78. doi:10.21427/q1a0-ny10

This Article is brought to you for free and open access by the School of Mathematics at ARROW@TU Dublin. It has been accepted for inclusion in Articles by an authorized administrator of ARROW@TU Dublin. For more information, please contact arrow.admin@tudublin.ie, aisling.coyne@tudublin.ie,gerard.connolly@tudublin.ie.

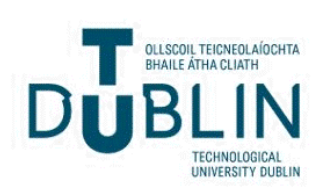




\title{
On Endomorphisms and Automorphisms of Some Torsion-Free Modules
}

\author{
BRENDAN GOLDSMITH \\ Abstract The relationship between the \\ endomorphism ring and the Automorphism group \\ of an Abelian p-group has been extensively \\ investigated. It is known that the \\ automorphism group need not generate the full \\ endomorphism ring. In this paper we \\ investigate the analagous problem for torsion- \\ free modules over a complete discrete \\ valuation ring obtaining similar results.
}

Some twenty of more years ago, L. Fuchs [4] raised

the question of the relationship between the automorphism group and the endomorphism ring of an Abelian p-group; in particular does the automorphism group generate the endomorphism ring? Fairly comprehensive answers were given in [1], [3], [7] and [9]. The object of this present work is to investigate the analogous problem for reduced torsion-free modules over a complete discrete valuation $r$ ing. We show in Theorem 7 , using a recent realization result on such modules [2], that the answer is, in general, in the negative. Throughout the work $\mathrm{R}$ will denote a complete discrete valuation ring i.e. a commutative principal ideal ring with exactly one prime element $p$ (up to unit factors) which is complete in the. topology having the ideals $\mathrm{p}^{\mathrm{n}} \mathrm{R}$ as a base of neighbourhoods of 0 . We shall always assume that 2 is a unit in $R$. If $G$ is a reduced torsion-free $\mathrm{R}$-module, then we shall denote the endomorphism 
418

\section{B. GOLDSMITH}

ring and automorphism group of $G$ by $E(G)$ and Aut $G$ respectively. $E_{0}(G)$ will denote the subring of $E(G)$ consisting of endomorphism of finite rank. Unexplained terms may be found in the standard works of Fuchs, [5], [6]; the main exception being that maps are written on the right.

We begin establishing that in some cases $E(G)$ is actually generated by Aut $G$; indeed the stronger result that in some of these cases every endomorphism is a sum of two automorphisms is established.

Proposition 1 If $M$ is a Eree $R$-module of finite rank, then every endomorphism of $M$ is a sum of two automorphisms of M.

\section{Proof}

$B y$ induction on the rank of $M$. The result is clearly true if $M$ has rank 1 . Suppose $M=R+G$ where rkG $=r k M-1$. If $\phi E E(M)$ then $\phi$ has a matrix representation of the form $\phi=\left[\begin{array}{ll}\mu & \alpha \\ \beta & \psi\end{array}\right]$ where $\mu \varepsilon E(R)$,

$\psi \varepsilon E(G)$ and $\alpha, \beta$ are appropriate homorphisms.

But by induction $\mu=\mu_{1}+\mu_{2}$ and $\psi=\psi_{1}+\psi_{2}$ where the $\mu_{i}, \psi_{i}$ are units. But then $\phi=\theta_{1}+\theta_{2}$ where

$$
\theta_{1}=\left[\begin{array}{ll}
\mu_{1} & \alpha \\
0 & \psi_{1}
\end{array}\right], \theta_{2}=\left[\begin{array}{ll}
\mu_{2} & 0 \\
\beta & \psi_{2}
\end{array}\right] \text {. }
$$

and it is easy to check that $\theta_{1}, \theta_{2}$ are

automorphisms.

\section{Proposition 2}

If $M$ is a countably generated reduced torsion-free module, then every endomorphism of $M$ is a sum of automorphisms of $M$. 


\section{Proof}

Since $M$ is countably generated it is free and we may write $M={ }_{i} \stackrel{\infty}{\stackrel{\oplus}{=}}\left\langle e_{i}\right\rangle$. Let $\phi \varepsilon E(M)$. Then for each $i$ we have $e_{i} \phi=\sum a_{i j} e_{j}$, where $a_{i j} \varepsilon R$ for all $i, j$. Since 2 is a unit in $R$, each element of $R$ can be expressed as a sum of two units and so, for each $i$, we may write $a_{i i}=b_{i i}+d_{i i}$ where $b_{i i}, d_{i i}$ are units in $\mathrm{R}$. Thus we may write

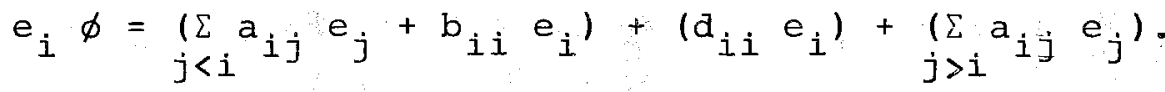

Hence $e_{i} \phi=e_{i} \phi_{1}+e_{i} \phi_{2}+e_{i} \phi_{3}$ where the endomorphisms $\phi_{i}$ are defined by the successive terms in the above expression. A simple check shows that $\phi_{1}$ and $\phi_{2}$ are automorphisms. The endomorphism $\phi_{3}$ given by $e_{i} \phi_{3}=\sum_{j>i} a_{i j} e_{j}$ is an $\alpha$-endomorphism in the terminology of Freedman [3] and so can be written as a sum of two locally nilpotent endomorphisms [3, Lemma 2]. Thus $\phi_{3}=\eta_{1}+\eta_{2}$ where each $\eta_{i}$ is locally nilpotent. But then $\phi_{3}=\left(n_{1}-1\right)+\left(1+n_{2}\right)$ where 1 is the identity endomorphism. However the local nilpotence of the $\eta_{i}$ ensure that $\left(\eta_{1}-1\right)$ and $\left(1+\eta_{2}\right)$ are units in $E(M)$. Thus $\phi$ is a sum of (four) automorphisms of $M$.

Corollary 3

If $M$ is a. free R-module, then every endomorphism of $M$ is a sum of automorphisms of $M$.

\section{Proof}

This follows from Proposition 2 by using an analogous set - theoretic construction to that given by Castagna [1, Theorem 2.2]. 
Lemma 4:

If $\mathrm{G}$ is a reduced torsion-free $\mathrm{R}$-module of infinite rank which is complete in its p-adic topology, then the Jacobson radical of $G, J(E(G)$ )

$=\mathrm{pE}(\mathrm{G})$.

Proof

See e.g. [8, Theorem 2.3].

Lemma 5:

Let $A$ be a ring with Jacobson radical $J(A)$. If each element of $A / J(A)$ is a sum of $n$ units, then each element of $A$ is a sum of $n$ units.

Proof

See e.g. [9, Lemma 2].

Proposition 6

If $G$ is a reduced torsion-free $R$-module of infinite rank which is complete in its p-adic topology, then every endomorphism of $G$ can be written as a sum of two automorphisms.

Proof Since $G$ is complete, $J(E(G))=\mathrm{pE}(G)$ and thus $E(G) / J(E(G))$ is a vector space over the field of $p$ elements, $\mathrm{Z}_{\mathrm{p}}$. But the results of Hill[7] clearly hold for such vector spaces and so each element of $E(G) / J(E(G))$ is a sum of two units. The result follows from Lemma 5 .

The results obtained in corollory 3 and Proposition 6 could be loosely interpreted as saying that at both extremities (in the sense that any reduced torsion-free $\mathrm{R}$-module can be regarded as lying between a free $R$-module and a complete $\mathrm{R}$-module) endomorphism rings are generated by the 
automorphism group in a strong fashion. Despite this, our next result shows that this does not always hold.

\section{Theorem 7}

There exists a reduced torsion-free R-module G such that the endomorphism ring of $G$ is not generated by the automorphism group of $G$. Proof

Let $A=R[x]$, the polynomial ring in a single variable $x$. It follows from a result of Dugas, Goldsmith and Gobel [2, Theorem 4.1] that there exists a reduced torsion-free $\mathrm{R}$-module $\mathrm{G}$ with $E(G)=A \oplus E_{0}(G)$. Define $\gamma: E(G) \rightarrow A \rightarrow A / p A=$ $z_{p}[x]$. The only units in $z_{p}[x]$ are non-zero constant polynomials and so the non-constant polynomials are not sums of units. Hence their pre-images under $\gamma$ are not sums of units.

Remark

It is not necessary to involve the full strength of theorem 4.1 in [2]. The simpler split realization result Theorem 0.1 in [2] will suffice but this was avoided here since the concept of inessential used there has no place in the current context.

Our final result shows that despite theorem 7 , the automorphism group generates a large part of the endomorphism ring.

Lemma 8:

If $\alpha$ is a finite rank endomorphism of the reduced torsion-free R-module $G$, then Ker $\alpha$ is a direct summand of $G$. Moreover there exists a direct 
decomposition $G=F \oplus K$ where $F$ has finite rank, $\mathrm{G} \alpha \subseteq \mathrm{F}$ and $\mathrm{K} \subseteq \mathrm{Ker} \alpha$.

Proof

This is a well known property of finite rank endomorphisms of torsion-free modules over a complete discrete valuation ring; see e.g.

[8, Lemma 3.2].

Proposition 9

If $G$ is a reduced torsion-free $R$-module and $\phi \varepsilon$ $E_{0}(G)$ then $\phi$ is a sum of two automorphisms.

\section{Proof}

From Lemma 8 we may write $G=F \oplus \mathrm{K}$ where $\mathrm{G} \phi \subseteq$ $F, K \subseteq \operatorname{ker} \phi$. Then $\phi \mid F$ is an endomorphism of $F$ which has finite rank and so, by proposition 1, $\phi \mid F=u+v$ where $u, v$ are automorphisms of $F$.

Now define endomorphisms $\mu, \nu$ of $G$ by:-

$$
\begin{aligned}
& f \mu=f u, k \mu=k \text { for } f \varepsilon F, k \varepsilon K, \\
& f \nu=f v, k \nu=-k \text { for } f \varepsilon F, k \varepsilon K .
\end{aligned}
$$

Clearly $\mu, v$ are units in $E(G)$ and moreover

$$
\begin{aligned}
& f(\mu+v)=f u+f v=f(u+v)=f \phi \\
& k(\mu+v)=k+(-k)=0=k \phi .
\end{aligned}
$$

Thus $\phi=\mu+\nu$ is a sum of two automorphisms.

\section{REFERENCES}

1. F. Castagna, Pacific J.Math 127(1968), 463-473 ouart.J.Math Oxford. (2), 35(1984), 131-146. 3. H. Freedman, J. London Math. Soc.

4. L. Fuchs, Topics in Abelian Groups

(Scott Foresman, 1963), 1 infinite abelian Groups, Vol. 1, Academic' $\frac{\text { Press, New York } 1970 .}{19}$ 
6. Infinite Abelian Groups, Vol. II, Academic Press, New York 1974.

7. P. Hill, Trans. amer. Math. Soc. 141 (1969), 99-105.

8. W. Liebert, Trans. Amer. Math. Soc. 169 (1972) 347-363.

9. R.W. Stringal1, Acta Math. Acad. Sci, Hungar. 18 (1967) 401-404. 